\title{
Association of dietary phytochemical index and mental health in women: a cross-sectional study
}

\author{
Manije Darooghegi Mofrad ${ }^{1,2}$, Fereydoun Siassi ${ }^{1}$, Bijan Guilani $^{3}$, Nick Bellissimo ${ }^{4}$ and \\ Leila Azadbakht ${ }^{1,5,6 *}$ \\ ${ }^{1}$ Department of Community Nutrition, School of Nutritional Sciences and Dietetics, Tehran University of Medical Sciences, PO \\ Box 1416643931, Tebran, Iran \\ ${ }^{2}$ Students' Scientific Research Center, Tehran University of Medical Sciences, PO Box 1417755331, Tehran, Iran \\ ${ }^{3}$ Department of Clinical Psychology, University of Tebran, Tehran, Iran \\ ${ }^{4}$ School of Nutrition, Ryerson University, Toronto, Ontario, Canada M5B $2 \mathrm{~K} 3$ \\ ${ }^{5}$ Diabetes Research Center, Endocrinology and Metabolism Clinical Sciences Institute, Tebran University of Medical Sciences, \\ PO Box 14155/6117, Tebran, Iran \\ ${ }^{6}$ Department of Community Nutrition, School of Nutrition and Food Science, Isfahan University of Medical Science, PO Box \\ 81745-151, Isfahan, Iran
}

(Submitted 3 September 2018 - Final revision received 3 January 2019 - Accepted 16 January 2019 - First published online 06 March 2019)

\section{Abstract}

Previous studies have shown that unhealthy dietary patterns are among the most important modifiable risk factors in the development of mental health disorders. We examined the association of dietary phytochemical index (DPI) with symptoms of depression, anxiety and psychological distress in Iranian women. In this cross-sectional study, a total of 488 women aged 20-50 years old attending health centres in the south of Tehran in 2018 were included. A validated and reliable FFQ was used for dietary assessment. Symptoms of depression, anxiety and psychological distress were assessed using a validated depression, anxiety, stress scales questionnaires with twenty-one-items. DPI was estimated using the following formula: (daily energy derived from phytochemical-rich foods $(\mathrm{kJ}) /$ total daily energy intake $(\mathrm{kJ})$ ) $\times 100$. The mean age of the study participants was 31.9 (SD 7.7) years. The prevalence of depressive symptoms, anxiety and psychological distress among study participants was $34 \cdot 6,40 \cdot 6$ and $42 \cdot 4 \%$, respectively. After controlling for potential confounders, women in the highest tertile of DPI had a lower prevalence of depressive symptoms (OR 0.22; $95 \%$ CI 0.12, 0.38) and anxiety (OR 0.33; $95 \%$ CI 0.20, 0.55), as well as psychological distress (OR 0.30; $95 \%$ CI 0.18, 0.49) compared with those in the lowest tertile. In conclusion, we found a significant association between DPI and mental health in women. Prospective studies are needed to confirm these findings.

Key words: Dietary phytochemical index: Diet quality: Depressive symptoms: Anxiety: Psychological distress

The most common mental disorders, depression and anxiety, are global health problems in both developed and developing countries $^{(1,2)}$. Major depression and anxiety affect about 4.7 and $7 \cdot 3 \%$ of people worldwide, respectively ${ }^{(1,3)}$. Reports from Iran suggest that about $21 \cdot 0$ and $20.8 \%$ of adults have anxiety and depressive symptoms, respectively ${ }^{(4)}$. On average, mental health disorders occur in Iranian women 1.7 times higher than men $(29 v .16 \%)^{(4)}$. Several studies have shown a significant association among chronic diseases, depression and anxiety $^{(5-7)}$. Since mental disorders impose a significant economic burden, and contribute to disability and premature death $^{(8,9)}$, it is important to find appropriate strategies to prevent these disorders.
A poor dietary pattern and physical inactivity are modifiable risk factors which may improve mental health outcomes ${ }^{(10,11)}$. Among the dietary factors, foods rich in phytochemical compounds including fruits, vegetables, nuts, legumes, soya, whole grains and olive oil have been associated with lower risk of mental health disorders ${ }^{(12-16)}$.

Phytochemicals are plant-based non-nutritive bioactive compounds including phenolic compounds (flavonoids, phenolic acids, hydroxycinammic acids, lignans, tyrosol esters, stilbenoids), isoperenoids, organosulphur compounds (allyl sulphurs, isothiocyanate $)^{(17-19)}$. Interestingly, intake of some of the phytochemicals have been associated with decreased risk of mortality ${ }^{(20)}, \mathrm{CVD}^{(21)}$, diabetes ${ }^{(22)}$ and several cancers ${ }^{(23)}$.

Abbreviations: DPI, dietary phytochemical index; SES, socio-economic status.

* Corresponding author: L. Azadbakht, fax +98 21 88984861, email azadbakhtleila@gmail.com 
Determining the amount of phytochemical compounds in the food supply or in human tissue samples is expensive, laborious and impractical for large populations. Therefore, alternative methods should be used to determine the phytochemical content of the foods. The dietary phytochemical index (DPI), which was presented for the first time by McCarty $^{(24)}$, is defined as the percentage of energy intake derived from phytochemical-rich foods. This index is an easy and practical method for the assessment of phytochemical intake and diet quality in clinical practice. Several epidemiologic studies have investigated the association between DPI and CVD risk factors including oxidative stress, obesity, blood pressure, blood sugar, lipid profile and cancer ${ }^{(25-33)}$. These studies showed the DPI was inversely related to obesity, oxidative stress, hypertriacylglycerolaemia, hypercholesterolaemia, insulin resistance, prediabetes, hypertension and breast cancer.

We are not aware of any publications regarding DPI and mental health. Therefore, this study aimed to determine the association between DPI and mental health in Iranian women.

\section{Methods}

\section{Study design and participants}

In this cross-sectional study, 488 women from ten health centres affiliated with the Tehran University of Medical Sciences (TUMS) were recruited using multistage cluster sampling. The study sample size was determined using the prevalence of mental disorders ${ }^{(4)}$ as the dependent variable, employing the formula: $N=\left[\left(Z_{1-\alpha} / 2\right)^{2} P(1-P)\right] / d^{2}$. Using $P=29, d=4.03$ and $\alpha=0.05$, we obtained 487 subjects. Women were included if they were between 20 and 50 years old, Iranian, not pregnant or lactating, not postmenopausal, not suffering from depression diagnosed by a psychiatrist or using anti-depressive drugs in the past 12 months, no history of an unfortunate events (such as severe financial problems, death of first-degree relatives or friends, love failure) in the past 6 months, not history of chronic diseases such as diabetes, cardiovascular, cancer, liver, kidney, pulmonary, thyroid, hypertension, epilepsy, multiple sclerosis disease, and were not following a specialised diet. In the current study, we excluded twenty-five participants whose total daily energy intake was outside the range of 2092-14644 kJ/d. Each participant provided informed written consent. In total, 488 participants were included in the statistical analysis. The study received ethics approval by the Medical Research Ethics Committee of TUMS.

\section{Dietary assessment}

Dietary intake was assessed using a 168-item semi-quantitative FFQ, which has been previously assessed for reliability and validity $^{(34)}$. All FFQ were completed through face-to-face interviews by a trained nutritionist. To convert the portion sizes of the consumed foods to grams, household measures were used. Mean energy and nutrient intakes from the FFQ were calculated using a modified version of NUTRITIONIST IV software for Iranian foods (version 7.0; N-Squared Computing).

\section{Phytochemical index calculation}

The DPI was determined based on method developed by McCarty; (DPI = (daily energy derived from phytochemical-rich foods $(\mathrm{kJ}) /$ total daily energy intake $(\mathrm{kJ})) \times 100)^{(24)}$. Fruits, vegetables, legumes, whole grains, nuts, soya products, seeds and olive oil were considered as phytochemical-rich foods. Potatoes were not included due to their poor source of phytochemicals. Natural fruit and vegetable juices as well as tomato sauces were considered in the fruit and vegetable groups due to their high phytochemical content.

\section{Assessment of psychological profile}

The psychological status of the participants was assessed by the validated depression, anxiety and stress scales (DASS-21) questionnaire $^{(35)}$. Each items of DASS-21 (depression, anxiety, psychological distress) contains seven questions. The answers were divided into four categories: zero, low, medium and high with a score between 0 and 3, respectively. DASS-21 is the summarised form of DASS-42. So, the total points for each item should be multiplied by 2 . Based on the total score, the subjects were ranked into five groups of normal (0-9), mild (10-13), moderate (14-20), severe (21-27) and very severe ( $>27$ ) for depressive symptoms. These descriptions for five groups of anxiety and stress were 0-7, 8-9, 10-14, 15-19, >20 and $0-14,15-18,19-25,26-33,>33$, respectively. However, due to the limited number of cases in some groups, they were divided into two groups of normal and mild/moderate/ severe/very severe. Depressive symptoms, anxiety and stress were defined as scores of equal or greater than 10, 8, and 15, respectively.

\section{Assessment of other variables}

The required information on other variables including age, marital status, socio-economic status (SES), smoking status, pregnancy or lactating, number of births, chronic diseases (diabetes, cardiovascular, cancer, liver, kidney, pulmonary, thyroid, hypertension, epilepsy, multiple sclerosis disease), family history of chronic disease, drug and supplement use, adherence to a specialised diet, menopause status, sleep duration, time away from home, stressful events in the past 6 months, and body size satisfaction was obtained from demographic questionnaires. SES score was computed as an index of SES based on family situation (head of family, self-care, under supervision), family size $(\leq 4,>4$ people), education ( $\leq$ diploma, >diploma), head of family education, occupation (housewife/unemployed/other, worker/free job, retired/ employee, manager), head of family job, number of employed member(s) of the family, number of rooms in the home, children under 18-year-old (yes/no), welfare status and amount of travel within and outside Iran (yes/no).

Body weight was measured to the nearest $0 \cdot 1 \mathrm{~kg}$, while participants wore minimal clothing and barefoot, using a digital scale (SECA). Height was measured to the nearest millimeter with an inflexible measuring rod. BMI calculated as weight in $\mathrm{kg}$ divided by height in $\mathrm{m}^{2}$. Physical activity was determined based 
on metabolic equivalents $\times \mathrm{h} / \mathrm{d}(\mathrm{MET}-\mathrm{h} / \mathrm{d})$ by recording physical activity over $24 \mathrm{~h}$ and the level of physical activity of individuals was calculated as MET-h/d ${ }^{(36)}$.

\section{Statistical analysis}

General characteristics across tertiles of DPI were expressed as means and standard deviations for continuous variables and numbers and percentages for categorical variables. To examine the differences across tertiles, we used an ANOVA for continuous variables and a $\chi^{2}$ test for categorical variables. Dietary intakes of study participants across tertiles of DPI were compared using ANCOVA and all values were adjusted for energy intake. We used binary logistic regressions to estimate OR and 95\% CI for psychological profile across tertiles of DPI in crude and multivariable-adjusted models. In these analyses, age, total energy intake, SES (low, medium and high), marital status (married, single), physical activity, supplement use (yes/ no), drug use (yes/no), family history of chronic disease (yes/ no), sleep duration, time away from home, body size image (normal, abnormal) and BMI were controlled in the adjusted model. $P_{\text {trend }}$ was determined by considering tertiles of DPI as ordinal variables in the logistic regression analysis. All statistical analyses were completed using the Statistical Package for Social Sciences (version 21; SPSS Inc.). $P<0.05$ was considered to be statistically significant.

\section{Results}

The general characteristics of the study participants across tertiles of DPI are presented in Table 1. Compared with those in the first tertile, the number of participants in the highest tertile of DPI were lower in the low SES level, and more likely to be married and were older. No other significant difference was seen in the general characteristics across tertiles of DPI.

The prevalence of symptoms of depression, anxiety and psychological distress were $34 \cdot 6,40 \cdot 6$ and $42 \cdot 4 \%$ of the study participants, respectively. The prevalence of depressive symptoms, anxiety and psychological distress across tertiles of DPI is shown in Fig. 1. Symptoms of depression, anxiety and psychological distress were significantly less prevalent among individuals in the top tertile of DPI compared with those in the bottom tertile.

Dietary intakes of study participants across tertiles of DPI are provided in Table 2. Participants in the top tertile had higher intakes of fruits, whole grain, vegetables, legumes, nuts, olive oil, Zn, K, Mg, vitamin C, vitamin E, vitamin A, total fibre, total carotenoids, carbohydrate $(P<0.001)$, seeds $(P=0.004)$ compared with those in the bottom tertile. Participants in the highest DPI consumed lower fat $(P=0 \cdot 02)$, PUFA $(P=0.03)$ and SFA $(P<0.001)$. In addition, there were no significant differences across tertiles of DPI for soya $(P=0.66)$, energy $(P=0 \cdot 81)$, protein $(P=0.08)$, MUFA $(P=0.54)$, cholesterol $(P=0 \cdot 13)$ and $\mathrm{Ca}(P=0 \cdot 11)$.

Crude and multivariable-adjusted OR (95\% CI) for mental health across tertiles of DPI for the whole population are presented in Table 3. After controlling for potential confounders, a significant association was observed between DPI and prevalence of symptoms of depression (OR 0.22; $95 \%$ CI $0.12,0.38 ; P<0.001$ ) and anxiety (OR $0.33 ; 95 \%$ CI 0.20 , $0.55 ; P<0.001$ ), as well as psychological distress (OR 0.30 ; $95 \%$ CI $0.18,0.49 ; P<0.001)$ among those in the highest $v$. lowest tertile of DPI.

\section{Discussion}

This cross-sectional study showed that the highest DPI was associated with decreased prevalence of symptoms of depression, anxiety and psychological stress in adult women.

Several studies have shown that plant foods are good sources to increase serum phytochemicals. Lipsky et al. ${ }^{(37)}$ successfully demonstrated whole plant foods (whole grains, vegetables, whole fruit, legumes, nuts, seeds) were positively related to serum carotenoids. Two studies showed serum carotenoids are considered a reliable biomarker of fruit and vegetable intake ${ }^{(38,39)}$. In fact, foods rich in phytochemicals are able to increase plasma phytochemicals after consumption. Therefore, DPI as food rich phytochemical score can be good indication of phytochemicals. Vincent et al. ${ }^{(26)}$ showed the DPI was positively correlated with the total carotenoid intake. In addition, this study showed that DPI decreased oxidative stress significantly. Phytochemicals were identified as antioxidant rich compounds. Therefore, decreasing oxidative stress could be a good reason that DPI evaluate the consumption of phytochemicals.

The value of the DPI can be judged from the range of interesting epidemiological studies that have shown that a highDPI diet is associated with a number of chronic disease. For example, several studies showed that DPI is inversely related to obesity, oxidative stress, hypertriacylglycerolaemia, hypercholesterolaemia, insulin resistance, hypertension, prediabetes and breast cancer ${ }^{(25-33)}$. This research is of importance, as it shows that diets in which a high proportion of energy content are provided by whole, phytochemical-rich plant foods can protect health. Although DPI is similar to other diet scores such as the prudent diet score and Mediterranean scores ${ }^{(40)}$ which were associated to mental health, these consist of a score inclusive of meats and fish or have some unhealthy components which gave reverse scores

Depression is a highly prevalent mental health disorder, which imposes a substantial burden to both individuals and society. It is expected to be the leading cause of disease and disability by the year $2030^{(41)}$. In this study, we found a significant inverse association between DPI and lower prevalence of depressive symptoms among Iranian women. Consistent with our findings, a previous meta-analysis showed dietary patterns characterised by high intakes of fruits, vegetables, whole grains, olive oil, fish, low-fat dairy products and antioxidants, and low content of animal foods have been associated with a lower odds of depression ${ }^{(40)}$. In addition, other studies showed that adherence to the Mediterranean diet (fruits, vegetables, cereals, nuts, olive oil, fish and wine) was associated with components of mental health ${ }^{(42)}$, particularly depression $^{(43)}$ and psychological resilience ${ }^{(44)}$. The common 
Table 1. General characteristics of participants across the tertiles (T) of dietary phytochemical index (DPI) (Mean values and standard deviations; numbers and percentages)

\begin{tabular}{|c|c|c|c|c|c|c|c|c|c|}
\hline \multirow[b]{3}{*}{ Variables } & & & \multicolumn{6}{|c|}{ DPI tertiles } & \multirow[b]{3}{*}{$P^{\star}$} \\
\hline & \multicolumn{2}{|c|}{ Total $(n 488)$} & \multicolumn{2}{|c|}{$\mathrm{T} 1 \leq 24(n 164)$} & \multicolumn{2}{|c|}{ T2 24 to $<32(n 164)$} & \multicolumn{2}{|c|}{$\mathrm{T} 3 \geq 32(n 160)$} & \\
\hline & Mean & SD & Mean & SD & Mean & SD & Mean & SD & \\
\hline Age (years) & 31.85 & 7.67 & 30.42 & 7.99 & $32 \cdot 17$ & 7.38 & 33.01 & 7.44 & 0.008 \\
\hline BMI $\left(\mathrm{kg} / \mathrm{m}^{2}\right)$ & 24.46 & 4.51 & 24.06 & 4.49 & $24 \cdot 60$ & 4.82 & 24.73 & $4 \cdot 18$ & 0.368 \\
\hline Weight (kg) & 64.44 & 11.99 & 63.49 & $11 \cdot 61$ & $64 \cdot 33$ & 12.45 & 65.52 & 11.88 & 0.309 \\
\hline Physical activity (MET-h/d) & $39 \cdot 9$ & $6 \cdot 65$ & $39 \cdot 16$ & $7 \cdot 10$ & 39.80 & $6 \cdot 40$ & $40 \cdot 76$ & $6 \cdot 35$ & 0.092 \\
\hline Sleeping time $(\mathrm{h})$ & $7 \cdot 78$ & 1.5 & $7 \cdot 80$ & 1.55 & 7.90 & 1.49 & $7 \cdot 63$ & 1.46 & 0.276 \\
\hline Out of home time $(\mathrm{h})$ & $6 \cdot 15$ & $3 \cdot 72$ & $6 \cdot 42$ & $3 \cdot 64$ & 5.92 & $3 \cdot 73$ & $6 \cdot 12$ & $3 \cdot 82$ & 0.48 \\
\hline \multicolumn{10}{|l|}{ Socio-economic status } \\
\hline \multicolumn{10}{|l|}{ Low } \\
\hline$n$ & \multicolumn{2}{|c|}{164} & \multicolumn{2}{|c|}{71} & & & & & 0.014 \\
\hline$\%$ & & & & & & & & & \\
\hline Medium & & & & & & & & & \\
\hline$n$ & & & & & & & & & \\
\hline$\%$ & & & & & & & & & \\
\hline High & & & & & & & & & \\
\hline$n$ & & & & & & & & & \\
\hline$\%$ & & & & & & & & & \\
\hline Overweight or obesity $\dagger$ & & & & & & & & & \\
\hline$n$ & & & & & & & & & 0.959 \\
\hline$\%$ & & & & & & & & & \\
\hline Marital status & & & & & & & & & \\
\hline Single & & & & & & & & & \\
\hline$n$ & & & & & & & & & 0.008 \\
\hline$\%$ & & & & & & & & & \\
\hline Married & & & & & & & & & \\
\hline$n$ & & & & & & & & & \\
\hline$\%$ & & & & & & & & & \\
\hline Education status & & & & & & & & & \\
\hline$\leq$ Diploma & & & & & & & & & \\
\hline$n$ & & & & & & & & & 0.324 \\
\hline$\%$ & & & & & & & & & \\
\hline > Diploma & & & & & & & & & \\
\hline$n$ & & & & & & & & & \\
\hline$\%$ & & & & & & & & & \\
\hline $\begin{array}{l}\text { Head of household educatio } \\
\leq \text { Diploma }\end{array}$ & & & & & & & & & \\
\hline$n$ & & & & & & & & & 0.364 \\
\hline$\%$ & & & & & & & & & \\
\hline > Diploma & & & & & & & & & \\
\hline$n$ & & & & & & & & & \\
\hline$\%$ & & & & & & & & & \\
\hline Supplement use & & & & & & & & & \\
\hline Yes & & & & & & & & & \\
\hline$n$ & & & & & & & & & 0.647 \\
\hline$\%$ & & & & & & & & & \\
\hline No & & & & & & & & & \\
\hline$n$ & & & & & & & & & \\
\hline$\%$ & & & & & & & & & \\
\hline Drug use & & & & & & & & & \\
\hline Yes & & & & & & & & & \\
\hline$n$ & & & & & & & & & 0.669 \\
\hline$\%$ & & & & & & & & & \\
\hline No & & & & & & & & & \\
\hline$n$ & & & & & & & & & \\
\hline$\%$ & & & & & & & & & \\
\hline Family history of chronic dis & & & & & & & & & \\
\hline Yes & & & & & & & & & \\
\hline$n$ & & & & & & & & & 0.386 \\
\hline$\%$ & & & & & & & & & \\
\hline No & & & & & & & & & \\
\hline$n$ & & & & & & & & & \\
\hline$\%$ & & & & & & & & & \\
\hline Body size satisfaction & & & & & & & & & \\
\hline Yes & & & & & & & & & \\
\hline$n$ & & & & & & & & & 0.272 \\
\hline$\%$ & & & & & & & & & \\
\hline
\end{tabular}


Table 1. Continued

\begin{tabular}{|c|c|c|c|c|c|c|c|c|c|}
\hline \multirow[b]{3}{*}{ Variables } & & & \multicolumn{6}{|c|}{ DPI tertiles } & \multirow[b]{3}{*}{$P^{*}$} \\
\hline & \multicolumn{2}{|c|}{ Total ( $n$ 488) } & \multicolumn{2}{|c|}{$\mathrm{T} 1 \leq 24(n 164)$} & \multicolumn{2}{|c|}{ T2 24 to $<32(n 164)$} & \multicolumn{2}{|c|}{$\mathrm{T} 3 \geq 32(n 160)$} & \\
\hline & Mean & SD & Mean & SD & Mean & SD & Mean & SD & \\
\hline \multicolumn{10}{|l|}{ No } \\
\hline$n$ & \multicolumn{2}{|c|}{161} & \multicolumn{2}{|c|}{59} & \multicolumn{2}{|c|}{57} & \multicolumn{2}{|c|}{45} & \\
\hline$\%$ & \multicolumn{2}{|c|}{33} & \multicolumn{2}{|c|}{37} & \multicolumn{2}{|c|}{35} & \multicolumn{2}{|c|}{28} & \\
\hline
\end{tabular}

MET, metabolic equivalents.

* Obtained from ANOVA for continuous variables and $x^{2}$ test for categorical variables.

$\dagger \mathrm{BMI} \geq 25 \mathrm{~kg} / \mathrm{m}^{2}$.

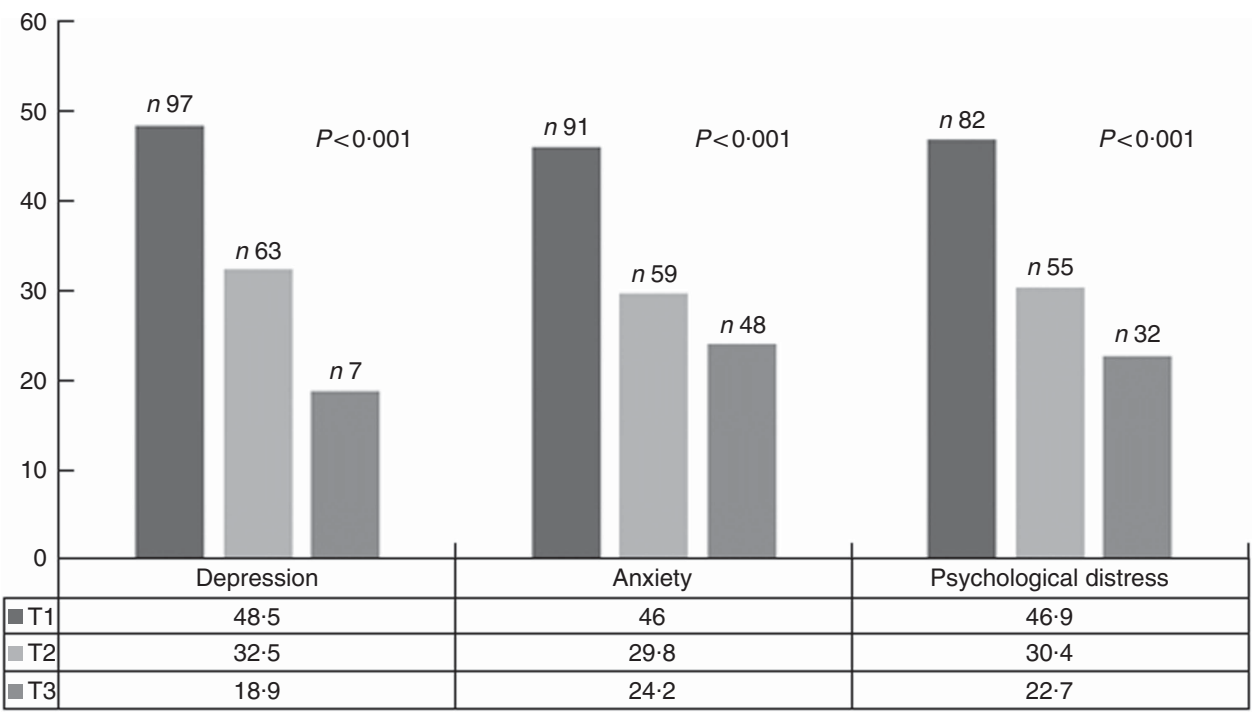

Fig. 1. Prevalence of depressive symptoms, anxiety and psychological distress across tertiles (T) of dietary phytochemical index. $\square$, T1; $\square$, T2; $\square$, T3.

characteristic of the aforementioned food groups is a wide range of phytochemicals with antioxidant and antiinflammatory properties. A cohort study showed a significant decreased risk of depressive symptoms among women with higher intakes of flavonoids ${ }^{(45)}$. In addition, epidemiological studies suggest that dietary polyphenols, such as flavonoids, phenolic acids and lignans may confer protection against depression $^{(46)}$. Moreover, one study found an inverse association between dietary polyphenol intake and depressive symptoms $^{(47)}$.

Anxiety are common disorders and are associated with elevated risk of $\mathrm{CHD}^{(48,49)}$. However, there has been less attention to the association between dietary patterns and anxiety. In the present study, we found that adherence to a higher DPI was associated with decreased anxiety. Previous studies have shown that vegetarian and traditional diets which included high phytochemical foods were associated to lower incidence of anxiety $^{(50,51)}$. Higher healthy diet scores (greater intake of vegetables, fruits, dairy products, nuts, olive oil and green olive, fish, legumes and whole grains) was associated with better mental health and lower anxiety ${ }^{(52)}$.

Psychological distress can increase the risk of stroke, myocardial infarction, ulcers and mental illnesses such as depression $^{(53)}$. We found that a higher DPI was related to decreased risk of psychological distress in women. In line with our finding, one study showed that consuming a Mediterranean diet was associated with a lower prevalence of psychological distress ${ }^{(54)}$.

Several possible mechanisms may explain the inverse association between DPI and mental health. Oxidative stress which is the imbalance between the production of reactive oxygen species and antioxidant defenses ${ }^{(55)}$ may contribute to the incidence of depression. The consumption of foods high in phytochemicals may decrease neuronal damage caused by oxidative stress ${ }^{(56-58)}$. Recent studies have shown some phytochemicals inhibit monoamine oxidase activity, mitochondrial enzymes that catalyse the oxidation of monoamines including serotonin, norepinephrine and dopamine have been associated with depression ${ }^{(59)}$. Phytochemicals could increase mRNA expression of pro-opiomelanocortin (a precursor of several peptides that seems to be dysregulated in depression). Phytochemicals have anti-inflammatory activity by decreasing biomarkers of inflammation, including TNF- $\alpha$ and IL-6, inhibiting neuronal growth especially in the hippocampus, and modification of gastrointestinal bacteria ratios ${ }^{(60,61)}$. Some studies demonstrated that inflammation involved in pathogenesis of mental health disorders ${ }^{(62)}$. Phytochemicals increase extracellular signal-regulated kinase phosphorylation, a main 
Table 2. Multivariable-adjusted dietary intakes across the tertiles (T) of dietary phytochemical index (DPI)* (Mean values with their standard errors)

\begin{tabular}{|c|c|c|c|c|c|c|c|}
\hline \multirow[b]{3}{*}{ Variables } & \multicolumn{6}{|c|}{ DPI tertile } & \multirow[b]{3}{*}{$P \dagger$} \\
\hline & \multicolumn{2}{|c|}{$\mathrm{T} 1 \leq 24(n 164)$} & \multicolumn{2}{|c|}{ T2 24 to <32 $(n 164)$} & \multicolumn{2}{|c|}{$\mathrm{T} 3 \geq 32(n 160)$} & \\
\hline & Mean & SE & Mean & $\mathrm{SE}$ & Mean & SE & \\
\hline Energy intake (kJ/d) & 8711.63 & $164 \cdot 26$ & 8651.96 & $164 \cdot 26$ & $8802 \cdot 21$ & $166 \cdot 31$ & 0.811 \\
\hline Protein $(\mathrm{g} / \mathrm{d})$ & 74.88 & $1 \cdot 13$ & 74.59 & 1.13 & 77.83 & $1 \cdot 14$ & 0.086 \\
\hline Carbohydrate (g/d) & 281.48 & 2.39 & 289.84 & 2.39 & 299.52 & $2 \cdot 42$ & $<0.001$ \\
\hline Fat $(g / d)$ & $78 \cdot 16$ & 1.04 & $76 \cdot 30$ & 1.04 & 74.07 & 1.05 & 0.023 \\
\hline SFA $(g / d)$ & 24.91 & 0.43 & 23.53 & 0.43 & $21 \cdot 31$ & 0.44 & $<0.001$ \\
\hline PUFA (g/d) & $17 \cdot 11$ & 0.39 & $16 \cdot 88$ & 0.39 & 15.73 & 0.39 & 0.031 \\
\hline MUFA (g/d) & $22 \cdot 89$ & 0.46 & $22 \cdot 23$ & 0.46 & $22 \cdot 29$ & 0.47 & 0.54 \\
\hline Cholesterol (mg/d) & 229.33 & $6 \cdot 16$ & $217 \cdot 07$ & $6 \cdot 16$ & $212 \cdot 41$ & $6 \cdot 24$ & 0.137 \\
\hline Vitamin A (RAE/d) & $1005 \cdot 21$ & $56 \cdot 84$ & 1321.07 & 56.85 & $1808 \cdot 91$ & 57.56 & $<0.001$ \\
\hline Vitamin $E(\mathrm{mg} / \mathrm{d})$ & 11.23 & 0.26 & $12 \cdot 34$ & 0.26 & $13 \cdot 16$ & 0.26 & $<0.001$ \\
\hline Vitamin $C(\mathrm{mg} / \mathrm{d})$ & $97 \cdot 13$ & $4 \cdot 15$ & $132 \cdot 81$ & 4.15 & 197.64 & $4 \cdot 21$ & $<0.001$ \\
\hline Total carotenoids $(\mu \mathrm{g} / \mathrm{d})$ & 546.9 & $54 \cdot 61$ & $860 \cdot 33$ & $54 \cdot 62$ & 1294.08 & $55 \cdot 30$ & $<0.001$ \\
\hline Total fibre $(\mathrm{g} / \mathrm{d})$ & 24.34 & 0.89 & 28.97 & 0.89 & $37 \cdot 12$ & 0.90 & $<0.001$ \\
\hline $\mathrm{Ca}(\mathrm{mg} / \mathrm{d})$ & $1019 \cdot 35$ & $22 \cdot 17$ & 1058.80 & $22 \cdot 17$ & $1085 \cdot 20$ & 22.45 & 0.111 \\
\hline $\mathrm{Mg}(\mathrm{mg} / \mathrm{d})$ & $236 \cdot 33$ & $3 \cdot 30$ & 264.82 & 3.30 & 304.91 & 3.34 & $<0.001$ \\
\hline $\mathrm{K}(\mathrm{mg} / \mathrm{d})$ & 2848.72 & 47.53 & 3380.43 & 47.53 & 4057.65 & $48 \cdot 13$ & $<0.001$ \\
\hline $\mathrm{Zn}(\mathrm{mg} / \mathrm{d})$ & 8.73 & 0.22 & 8.69 & 0.22 & 9.74 & 0.22 & 0.001 \\
\hline Fruit (g/d) & $193 \cdot 18$ & $13 \cdot 28$ & $290 \cdot 37$ & $13 \cdot 28$ & $448 \cdot 33$ & 13.44 & $<0.001$ \\
\hline Vegetable $(g / d)$ & $250 \cdot 26$ & $13 \cdot 13$ & 347.63 & $13 \cdot 13$ & 475.85 & $13 \cdot 29$ & $<0.001$ \\
\hline Nuts $(g / d)$ & 4.84 & 0.88 & 7.98 & 0.88 & $13 \cdot 72$ & 0.89 & $<0.001$ \\
\hline Legumes $(\mathrm{g} / \mathrm{d})$ & $34 \cdot 11$ & $2 \cdot 7$ & 43.95 & 2.74 & 50.93 & 2.78 & $<0.001$ \\
\hline Whole grains $(g / d)$ & $7 \cdot 12$ & 0.97 & 9.96 & 0.98 & $14 \cdot 17$ & 0.99 & $<0.001$ \\
\hline Seeds (g/d) & $5 \cdot 34$ & 0.74 & $6 \cdot 92$ & 0.74 & $8 \cdot 27$ & 0.84 & 0.004 \\
\hline Olive oil (g/d) & 1.29 & 0.29 & $2 \cdot 29$ & 0.29 & 4.44 & 0.30 & $<0.001$ \\
\hline Soya sources $(\mathrm{g} / \mathrm{d})$ & $4 \cdot 12$ & 0.59 & $4 \cdot 13$ & 0.59 & 4.79 & 0.60 & 0.663 \\
\hline
\end{tabular}

RAE, retinol activity equivalents.

${ }^{*}$ All values are adjusted for energy intake, except for total energy.

†Obtained from ANCOVA.

Table 3. Symptoms of depression, anxiety and psychological distress across tertiles ( $T$ ) of dietary phytochemical index (DPI)

(Multivariable-adjusted odds ratios and $95 \%$ confidence intervals)

\begin{tabular}{|c|c|c|c|c|c|c|}
\hline \multirow[b]{3}{*}{ Variables } & \multicolumn{5}{|c|}{ DPI tertiles } & \multirow[b]{3}{*}{$P_{\text {trend }}$} \\
\hline & \multirow[b]{2}{*}{$\mathrm{T} 1 \leq 24(n 164)$} & \multicolumn{2}{|c|}{ T2 24 to <32 $(n 164)$} & \multicolumn{2}{|c|}{$\mathrm{T} 3 \geq 32(n 160)$} & \\
\hline & & OR & $95 \% \mathrm{Cl}$ & OR & $95 \% \mathrm{Cl}$ & \\
\hline \multicolumn{7}{|c|}{ Depressive symptoms } \\
\hline Crude & 1.00 & 0.50 & $0.32,0.78$ & 0.25 & $0.15,0.41$ & $<0.001$ \\
\hline Adjusted* & 1.00 & 0.47 & $0.29,0.76$ & 0.22 & $0.12,0.38$ & $<0.001$ \\
\hline \multicolumn{7}{|l|}{ Anxiety } \\
\hline Crude & 1.00 & 0.45 & $0.28,0.70$ & 0.34 & $0.21,0.54$ & $<0.001$ \\
\hline Adjusted & 1.00 & 0.45 & $0.28,0.72$ & 0.33 & $0.20,0.55$ & $<0.001$ \\
\hline \multicolumn{7}{|c|}{ Psychological distress } \\
\hline Crude & 1.00 & 0.43 & $0.27,0.67$ & 0.28 & $0.18,0.45$ & $<0.001$ \\
\hline Adjusted & 1.00 & 0.45 & $0.28,0.72$ & 0.30 & $0.18,0.49$ & $<0.001$ \\
\hline
\end{tabular}

*Adjusted for age, energy intake, socio-economic status, physical activity, marriage status, supplement use, drug use, family history of chronic disease, satisfaction with the physical form, number of sleeping hours, and number of outside hours, BMI.

† Obtained from logistic regression by considering tertiles of DPI as ordinal variable.

component of the neurotrophic and specific signaling pathways participating in neuronal differentiation and plasticity ${ }^{(63)}$. Phytochemicals also have been shown to affect multiple neurotrophic and neuroprotective mechanisms ${ }^{(64)}$.

This study has some limitations. First, DPI was estimated by the amount of energy provided from foods rich in phytochemicals. However, some foods with high phytochemical content such as green and black tea were not considered because they did not contribute energy. Second, we did not take into account the type of phytochemicals consumed, therefore, the quality of dietary phytochemicals may have differed among people with the same DPI score resulting in a more varied effect of mental health. Third, the cross-sectional study design cannot show causal link between DPI and mental health. Fourth, our study assessed only symptoms of depression and anxiety, but not the diagnosis of these disorders. Fifth, 
although we attempted to control for known confounding factors, there may have been some residual confounding. Finally, the study was performed only in females aged 20-50 years, which affects the generalisability to the larger population.

In conclusion, we found that higher DPI was associated with a lower risk of depressive symptoms, anxiety and psychological distress. These initial findings need to be confirmed with prospective studies to confirm the relationship between DPI and mental health.

\section{Acknowledgements}

The study was financially supported by TUMS.

This study was extracted from a dissertation that was approved by TUMS (no. 9511468003).

M. D. M., F. S., B. G. and L. A. contributed to conception, design, data collection, statistical analyses, data interpretation, manuscript drafting and approval of the final version of the manuscript and agreed for all aspects of the work. N. B. revised the manuscript and contributed to data interpretation.

None of the authors had any personal or financial conflicts of interest.

\section{References}

1. Baxter A, Scott K, Vos T, et al. (2013) Global prevalence of anxiety disorders: a systematic review and meta-regression. Psychol Med 43, 897-910.

2. Steel Z, Marnane C, Iranpour C, et al. (2014) The global prevalence of common mental disorders: a systematic review and meta-analysis 1980-2013. Int J Epidemiol 43, 476-493.

3. Ferrari A, Somerville A, Baxter A, et al. (2013) Global variation in the prevalence and incidence of major depressive disorder: a systematic review of the epidemiological literature. Psychol Med 43, 471-481.

4. Noorbala A, Yazdi SB, Yasamy M, et al. (2004) Mental health survey of the adult population in Iran. BrJ Psychiatry 184, 70-73.

5. Vaccarino V, McClure C, Johnson BD, et al. (2008) Depression, the metabolic syndrome and cardiovascular risk. Psychosom Med 70, 40-48.

6. Pan A, Keum N, Okereke OI, et al. (2012) Bidirectional association between depression and metabolic syndrome: a systematic review and meta-analysis of epidemiological studies. Diabetes Care 35, 1171-1180.

7. van Reedt Dortland AK, Giltay EJ, Van Veen T, et al. (2010) Metabolic syndrome abnormalities are associated with severity of anxiety and depression and with tricyclic antidepressant use. Acta Psychiatr Scand 122, 30-39.

8. Olesen J, Gustavsson A, Svensson M, et al. (2012) The economic cost of brain disorders in Europe. Eur J Neurol 19, 155-162.

9. Sobocki P, Jönsson B, Angst J, et al. (2006) Cost of depression in Europe. J Ment Health Policy Econ 9, 87-98.

10. Popa T \& Ladea M (2012) Nutrition and depression at the forefront of progress. J Med Life 5, 414-419.

11. Quirk SE, Williams LJ, O'Neil A, et al. (2013) The association between diet quality, dietary patterns and depression in adults: a systematic review. BMC Psychiatry 13, 175.

12. Hosseinzadeh M, Vafa M, Esmaillzadeh A, et al. (2016) Empirically derived dietary patterns in relation to psychological disorders. Public Health Nutr 19, 204-217.

13. Yu S, Guo X, Yang H, et al. (2015) Soybeans or soybean products consumption and depressive symptoms in older residents in rural Northeast China: a cross-sectional study. J Nutr Health Aging 19, 884-893.

14. Akbaraly TN, Brunner EJ, Ferrie JE, et al. (2009) Dietary pattern and depressive symptoms in middle age. Br J Psychiatry 195, 408-413.

15. Lang UE, Beglinger C, Schweinfurth N, et al. (2015) Nutritional aspects of depression. Cell Physiol Biochem 37, 1029-1043.

16. Su Q, Yu B, He H, et al. (2016) Nut consumption is associated with depressive symptoms among Chinese adults. Depress Anxiety 33, 1065-1072.

17. Han X, Shen T \& Lou H (2007) Dietary polyphenols and their biological significance. Int J Mol Sci 8, 950-988.

18. Vazquez-Prieto MA \& Miatello RM (2010) Organosulfur compounds and cardiovascular disease. Mol Aspects Med 31, 540-545.

19. Tucci SA (2010) Phytochemicals in the control of human appetite and body weight. Pharmaceuticals 3, 748-763.

20. Grosso G, Micek A, Godos J, et al. (2017) Dietary flavonoid and lignan intake and mortality in prospective cohort studies: systematic review and dose-response meta-analysis. Am J Epidemiol 185, 1304-1316.

21. Wang X, Ouyang YY, Liu J, et al. (2014) Flavonoid intake and risk of CVD: a systematic review and meta-analysis of prospective cohort studies. Br J Nutr 111, 1-11.

22. Liu YJ, Zhan J, Liu XL, et al. (2014) Dietary flavonoids intake and risk of type 2 diabetes: a meta-analysis of prospective cohort studies. Clin Nutr 33, 59-63.

23. Grosso G, Godos J, Lamuela-Raventos R, et al. (2017) A comprehensive meta-analysis on dietary flavonoid and lignan intake and cancer risk: level of evidence and limitations. Mol Nutr Food Res 61, 1600930.

24. McCarty MF (2004) Proposal for a dietary "phytochemical index". Med Hypotheses 63, 813-817.

25. Bahadoran Z, Golzarand M, Mirmiran P, et al. (2013) The association of dietary phytochemical index and cardiometabolic risk factors in adults: Tehran Lipid and Glucose Study. J Hum Nutr Diet 26, 145-153.

26. Vincent HK, Bourguignon CM \& Taylor AG (2010) Relationship of the dietary phytochemical index to weight gain, oxidative stress and inflammation in overweight young adults. J Hum Nutr Diet 23, 20-29.

27. Golzarand M, Bahadoran Z, Mirmiran P, et al. (2015) Dietary phytochemical index is inversely associated with the occurrence of hypertension in adults: a 3-year follow-up (the Tehran Lipid and Glucose Study). Eur J Clin Nutr 69, 392-398.

28. Mirmiran P, Bahadoran Z, Golzarand M, et al. (2012) Association between dietary phytochemical index and 3-year changes in weight, waist circumference and body adiposity index in adults: Tehran Lipid and Glucose study. Nutr Metab 9, 108 .

29. Bahadoran Z, Karimi Z, Houshiar-rad A, et al. (2013) Dietary phytochemical index and the risk of breast cancer: a case control study in a population of Iranian women. Asian Pac J Cancer Prev 14, 2747-2751.

30. Bahadoran Z, Mirmiran P, Tohidi M, et al. (2015) Dietary phytochemical index and the risk of insulin resistance and beta-cell dysfunction: a prospective approach in Tehran Lipid and Glucose Study. Int J Food Sci Nutr 66, 950-955.

31. Golzarand M, Mirmiran P, Bahadoran Z, et al. (2014) Dietary phytochemical index and subsequent changes of lipid profile: a 3-year follow-up in Tehran Lipid and Glucose Study in Iran. ARYA Atheroscler 10, 203-210.

32. Carnauba RA, Chaves DF, Baptistella AB, et al. (2017) Association between high consumption of phytochemical-rich foods and anthropometric measures: a systematic review. Int J Food Sci Nutr 68, 158-166. 
33. Abshirini M, Mahaki B, Bagheri F, et al. (2018) Higher intake of phytochemical-rich foods is inversely related to prediabetes: a case-control study. Int J Prev Med 9, 64.

34. Azadbakht L, Mirmiran P, Esmaillzadeh A, et al. (2005) Dairy consumption is inversely associated with the prevalence of the metabolic syndrome in Tehranian adults. Am J Clin Nutr 82, 523-530.

35. Henry JD \& Crawford JR (2005) The short-form version of the Depression Anxiety Stress Scales (DASS-21): construct validity and normative data in a large non-clinical sample. $\mathrm{Br} \mathrm{J} \mathrm{Clin}$ Psychol 44, 227-239.

36. Seyed GR, Gholi Z, Saraf BS, et al. (2011) Fruit and vegetable intake associated with body mass index and waist circumference in young female students. J Educ Health Promot 7, 35-42.

37. Lipsky LM, Cheon K, Nansel TR, et al. (2012) Candidate measures of whole plant food intake are related to biomarkers of nutrition and health in the US population (National Health and Nutrition Examination Survey 1999-2002). Nutr Res 32, 251-259.

38. van Kappel AL, Steghens JP, Zeleniuch-Jacquotte A, et al. (2001) Serum carotenoids as biomarkers of fruit and vegetable consumption in the New York Women's Health Study. Public Health Nutr 4, 829-835.

39. Institute of Medicine (US) Panel on Dietary Antioxidants and Related Compounds (2000) Dietary Reference Intakes for Vitamin C, Vitamin E, Selenium and Carotenoids. A Report of the Panel on Dietary Antioxidants and Related Compounds, Subcommittees on Upper Reference Levels of Nutrients and of Interpretation and Use of Dietary Reference Intakes and the Standing Committee on the Scientific Evaluation of Dietary Reference Intakes, Food and Nutrition Board, Institute of Medicine. Washington, DC: National Academies Press.

40. Li Y, Lv M-R, Wei Y-J, et al. (2017) Dietary patterns and depression risk: a meta-analysis. Psychiatry Res 253, 373-382.

41. Payne ME, Steck SE, George RR, et al. (2012) Fruit, vegetable, and antioxidant intakes are lower in older adults with depression. J Acad Nutr Diet 112, 2022-2027.

42. Muros JJ, Cofre-Bolados C, Arriscado D, et al. (2017) Mediterranean diet adherence is associated with lifestyle, physical fitness, and mental wellness among 10-y-olds in Chile. Nutrition 35, 87-92.

43. Sánchez-Villegas A, Martínez-González MA, Estruch R, et al. (2013) Mediterranean dietary pattern and depression: the PREDIMED randomized trial. BMC Med 11, 208.

44. Bonaccio M, Di Castelnuovo A, Costanzo S, et al. (2018) Mediterranean-type diet is associated with higher psychological resilience in a general adult population: findings from the Moli-sani study. Eur J Clin Nutr 72, 154-160.

45. Chang SC, Cassidy A, Willett WC, et al. (2016) Dietary flavonoid intake and risk of incident depression in midlife and older women. Am J Clin Nutr 104, 704-714.

46. Sureda A \& Tejada S (2015) Polyphenols and depression: from chemistry to medicine. Curr Pharm Biotechnol 16, 259-264.

47. Godos J, Castellano S, Ray S, et al. (2018) Dietary polyphenol intake and depression: results from the Mediterranean Healthy Eating, Lifestyle and Aging (MEAL) study. Molecules 23, 999.
48. Kubzansky LD, Kawachi I, Weiss ST, et al. (1998) Anxiety and coronary heart disease: a synthesis of epidemiological, psychological, and experimental evidence. Ann Behav Med 20, 47-58.

49. Albert CM, Chae CU, Rexrode KM, et al. (2005) Phobic anxiety and risk of coronary heart disease and sudden cardiac death among women. Circulation 111, 480-487.

50. Jacka FN, Pasco JA, Mykletun A, et al. (2010) Association of Western and traditional diets with depression and anxiety in women. Am J Psychiatry 167, 305-311.

51. Yannakoulia M, Panagiotakos DB, Pitsavos C, et al. (2008) Eating habits in relations to anxiety symptoms among apparently healthy adults. A pattern analysis from the ATTICA study. Appetite 51, 519-525.

52. Jacka FN, Mykletun A, Berk M, et al. (2011) The association between habitual diet quality and the common mental disorders in community-dwelling adults: the Hordaland Health study. Psychosom Med 73, 483-490.

53. Sapolsky RM (2004) Why Zebras Don't Get Ulcers: The Acclaimed Guide to Stress, Stress-related Diseases, and Coping - Now Revised and Updated. New York: St Martin's Press.

54. Hodge A, Almeida OP, English DR, et al. (2013) Patterns of dietary intake and psychological distress in older Australians: benefits not just from a Mediterranean diet. Int Psychogeriatr 25, 456-466

55. Birben E, Sahiner UM, Sackesen C, et al. (2012) Oxidative stress and antioxidant defense. World Allergy Organ J 5, 9-19.

56. Gougeon L (2014) Nutritional predictors of depression in a cohort of community-dwelling elderly Canadians: NuAge cohort. Appl Physiol Nutr Metab 39, 1412.

57. Ibarra O, Gili M, Roca M, et al. (2015) The Mediterranean diet and micronutrient levels in depressive patients. Nutr Hosp $\mathbf{3 1}$, 1171-1175.

58. Popa TA \& Ladea M (2012) Nutrition and depression at the forefront of progress. J Med Life 5, 414-419.

59. Grosso C, Valentão P, Ferreres F, et al. (2013) The use of flavonoids in central nervous system disorders. Curr Med Chem 20, 4694-4719.

60. Dash S, Clarke G, Berk M, et al. (2015) The gut microbiome and diet in psychiatry: focus on depression. Curr Opin Psychiatry 28, 1-6.

61. Rendeiro C, Rhodes JS \& Spencer JP (2015) The mechanisms of action of flavonoids in the brain: direct $v$. indirect effects. Neurochem Int 89, 126-139.

62. Howren MB, Lamkin DM \& Suls J (2009) Associations of depression with C-reactive protein, IL-1, and IL-6: a metaanalysis. Psychosom Med 71, 171-186.

63. Xiong Z, Jiang B, Wu P-F, et al. (2011) Antidepressant effects of a plant-derived flavonoid baicalein involving extracellular signal-regulated kinases cascade. Biol Pharm Bull 34, 253-259.

64. Bahramsoltani R, Farzaei MH, Farahani MS, et al. (2015) Phytochemical constituents as future antidepressants: a comprehensive review. Rev Neurosci 26, 699-719. 